\title{
The approach to diamond growth on levitating seed particles
}

\author{
Satoshi Shimizu ${ }^{1 *}$, Tetsuji Shimizu ${ }^{1}$, Beatrice M. Annaratone ${ }^{1}$, \\ Wolfgang Jacob ${ }^{2}$, Hubertus Thomas ${ }^{1}$, Noriyoshi Sato ${ }^{1,3}$, and Gregor E. Morfill ${ }^{1}$ \\ ${ }^{1}$ Max-Planck-Institut für extraterrestrische Physik, \\ Gießenbachstraße, D-85748 Garching, Germany \\ ${ }^{2}$ Max-Planck-Institut für Plasmaphysik \\ Boltzmannstraße 2, D-85748 Garching, Germany \\ ${ }^{3}$ Professor Emeritus, Tohoku University, Sendai 980-8579, Japan
}

\begin{abstract}
We demonstrate the approach of diamond growth on levitating seed particles in a rf plasma. We introduce a hot filament chemical vapor deposition (CVD) technique into the rf plasma chamber in order to obtain improved crystal growth. Firstly, we confirmed diamond nucleation on seed particles placed on a Si substrate using the hot filament CVD. The deposition conditions, namely the total pressure and the rf power, were chosen so that they correspond to particles levitation conditions. We observe that a hydrogen pre-treatment on the seed particles improves the nucleation. Secondly, we confirm the levitation of particles at high temperatures. Fine particles levitated in a plasma are particularly sensitive to thermophoretic effects due to inhomogeneities in the gas heating. Therefore, proper heating procedures are required for successful particles levitation.
\end{abstract}

PACS: 52.27.Lw, 81.05.Uw, 81.15.Gh

Keywords: diamond, CVD, hot filament, particle, levitation

* corresponding autor, Tel: +49-89-300003016, Fax: +49-89-300003399

Email address: sshimizu@,mpe.mpg.de (S. Shimizu).

doi:10.1016/j.apsusc.2007.07.017 


\section{Introduction}

Fine particles of diameters few micrometers in a plasma are negatively charged and sensitive to the balance of several forces, e.g., gravity, electrostatic, thermophoretic, ion drag and others $[1,2]$. Therefore, choosing the proper plasma parameters allows to levitate particles at a plasma sheath region. The motion of levitated particles is not always random but, for example, Coulomb crystals cloud form at certain parameters [3]. Levitation conditions strongly depend on the particles size and shape [4] as well as on the type of gas. Here, successful fine diamond particles levitation in a hydrogen plasma have been observed in hydrogen plasmas, although their non-spherical shapes could cause a disturbance in the levitation due to the anisotropic forces [5]. This leads to the possibility of homoepitaxial growth of diamond on the levitated particles in a $\mathrm{CH}_{4} / \mathrm{H}_{2}$ plasma. This is the deposition not on a planar substrate (two-dimensional, 2-D) but on levitating particles (3-D) and it is technically interesting. In our previous study, we succeeded in observing island growth of carbon materials on levitated seed particles by using a radio frequency (rf) plasma CVD [6]. Our next step is to enhance the growth rate with maintaining diamond crystallinity. In order to obtain high crystallinity CVD diamonds, one needs: 1) high density of atomic hydrogen and 2) effective heating of the substrate (particles in our case). To accomplish these demands, we introduce a hot filament CVD technique into a rf plasma, with which one can expect high density of atomic hydrogen as well as precursors than those in a rf plasma at a

particles levitation condition (e.g., electron density $\sim 10^{9} \mathrm{~cm}^{-3}$ ) [7-10]. Additionally, effective heating of levitated particles are expected due to a heat radiation from the filament. In this paper, we report the results of diamond growth using HFCVD, as well as fine particles levitation in a rf plasma chamber equipped with a hot filament.

\section{Experimental}

Figure 1 (a) shows the schematic of the HFCVD system. A Si substrate is placed on the ceramic heater to which a thermocouple is connected. Approximately $1.5 \mathrm{~cm}$ above the substrate, a tungsten filament $(\phi=0.3 \mathrm{~mm})$ is mounted. The temperature of the filament is monitored by a pyrometer. The typical base pressure is $\approx 2 \times 10^{-4} \mathrm{~Pa}$. Prior to the deposition, filament baking and carbide processes were carried out. These treatments were performed without diamond particles in order to avoid possible contaminations on the particles 
surfaces. After the procedures, the diamond fine particles $(\phi=2-4 \mu \mathrm{m})$ stored in a dispenser are spread over the substrate. Then, the particles surfaces are treated with atomic hydrogen formed by the hot filament before starting a diamond deposition. The fixed deposition parameters are: total pressure $=100 \mathrm{~Pa}$, filament temperature $=2000{ }^{\circ} \mathrm{C}$, substrate temperature $=750{ }^{\circ} \mathrm{C}, \mathrm{CH}_{4}$ flow rate $=1 \mathrm{sccm}, \mathrm{H}_{2}$ flow rate $=100 \mathrm{sccm}$ and deposition time $=3 \mathrm{~h}$. After the deposition, the resulting particles were observed by the scanning electron microscope (SEM) (Philips, XL 30 ESEM) and micro-Raman spectroscopy (WITec, CRM 200).

Figure 1 (b) shows the schematic of the particles levitation system. The experiment was carried out in parallel-plate capacitively coupled rf plasmas. The bottom electrode is driven by $\mathrm{rf}$ and the top electrode is grounded. The distance between the electrodes is $12 \mathrm{~cm}$ and their diameters are $10 \mathrm{~cm}$. After the plasma is ignited between the electrodes, diamond fine particles are supplied to the plasma from the dispenser. On the bottom electrode, a particle confinement ring is placed to modify sheath electric field so that the particles are confined above the inside region of the ring. An Ar ion laser is equipped to the system for illuminating the levitated particles. The particles motions are monitored by a CCD camera which detects the scattered laser light from the levitated particles.

\section{Resuts and discussion}

\subsection{HFCVD on diamond seed particles}

Using the HFCVD, we check diamond growth on seed particles placed on a Si substrate. Therefore, this is not a 3-D but 2-D deposition. The purpose of the experiments is to find out the suitable deposition parameters with respect to the levitation conditions. For example, a high pressure condition generally results in high growth rate, however, it makes the thickness of plasma sheath thin, which results in a difficulty in particles levitation. In this experiment, we choose the total pressure of $100 \mathrm{~Pa}$ which is suitable for levitation.

A surface pre-treatment process is important for achieving successful CVD-depositions. We chose a dry process for the easy handling of fine particles. The depositions were carried out at the same conditions but at different hydrogen pre-treatment times. Figure 2 (a) is the image of the initial seed particles for comparison (without deposition), and Figures 2 (b), (c) and (d) are the images of the samples prepared with the hydrogen pre-treatment time of 
0,1 and $3 \mathrm{~h}$, respectively. We observe that the number of nucleation sites increases with the pre-treatment time.

Generally, surface reactions in diamond growth are considered like below $[11,12]$.

$\mathrm{H}+\mathrm{H}-\mathrm{C}_{\mathrm{D}} \rightarrow \mathrm{C}_{\mathrm{D}} *+\mathrm{H}_{2}$,

$\mathrm{CH}_{n}+\mathrm{C}_{\mathrm{D}} * \rightarrow \mathrm{H}_{n} \mathrm{C}-\mathrm{C}_{\mathrm{D}}$,

where $\mathrm{H}-\mathrm{C}_{\mathrm{D}}$ is a hydrogen terminated diamond surface, $\mathrm{C}_{\mathrm{D}}{ }^{*}$ is a diamond surface with a dangling bond and $\mathrm{CH}_{n}$ is a precursor. In reaction (1), the surface terminating hydrogen is abstracted by a gaseous phase atomic hydrogen, remaining a surface dangling bond. Then, at a certain probability, a precursor reacts with it and forms a new $\mathrm{sp}^{3}$ bond. We have detected the surface oxygen peak in our seed particles by X-ray Photoelectron Spectroscopy (XPS). Moreover, Goeting et al. observed the decrease in the $\mathrm{O} 1 \mathrm{~s} / \mathrm{C} 1 \mathrm{~s}$ signals ratio by exposing the diamond surface to a hydrogen plasma [13]. We speculate here that the hydrogen treatment passivates the native oxygen layers of seed particles surfaces and creates hydrogen-terminated layers, resulting in a preferable surface for diamond growth. Further investigations are necessary to understand the effect.

Next, the samples were observed by micro-Raman spectroscopy to confirm the diamond growth. Figure 3 shows the spectra of the samples prepared with the pre-treatment time of 0 , 1 and $3 \mathrm{~h}$. The spectrum of the seed particles is also shown for comparison (without deposition). Each spectrum shows the clear peak around $1332 \mathrm{~cm}^{-1}$ which is an active mode of diamond. Here, it is necessary to distinguish the diamond peaks from those of the seed particles. For this, we pay attention to the observed peak shifts and compare them in Figure 4 in which the peaks of the reference seed particles and of the $3 \mathrm{~h}$ pre-treated sample are shown. For comparison, the peak of the diamond (100) substrate is also shown. The micro-Raman spectra were taken by planner scan with $100 \times 100$ dots and the peaks at maximum and minimum positions among others are shown. The peak shift range of the reference particles is $1328-1333 \mathrm{~cm}^{-1}$ and that of the diamond deposited particles is 1315 $-1320 \mathrm{~cm}^{-1}$. We observe the lower frequencies peaks in the diamond deposited particles. Shifts of diamond $\mathrm{sp}^{3}$ peak have been discussed from several perspectives, e.g., residual stress, phonon confinement and defect scattering. As for stress, the values are estimated according to a type of stress and its direction with respect to a crystal orientation [14]. The diameters of the deposited materials are different from those of the seed particles (Fig. 2 (d)). If we assume a grain size effect on residual stress, one can distinguish a peak of 
deposited diamond from that of reference particles by observing its shift. Ager III et al. calculated the peak shifts and the change in full width at half maximum (FWHM) based on phonon confinement model where those phenomena are related to a phonon confinement length [15]. They summarized that a lower peak position with broader FWHM is obtained in a shorter confinement length condition assuming an averaged (100) phonon-dispersion curve. In Fig. 4, we detect the peak shifts as well as the FWHM changes (the diamond (100) substrate: $4 \mathrm{~cm}^{-1}$, the reference particles: $5 \mathrm{~cm}^{-1}$ and the diamond deposited particles: $8 \mathrm{~cm}^{-1}$ ). The absolute values of peak shifts and FWHM changes with respect to the crystal sizes do not correspond to those in the literature, but the trends are similar. Moreover, Nachal'naya et al. observed the relation between the peak shift and the densities of the particles surface defects [16]. They observed that both the peak shift and the defect density correspond to the diamond powder size. Note, that their spectra also show FWHM changes. To confirm this effect further, it is necessary to measure the defect densities of our samples. Assuming a crystal size effect on Raman spectrum peak position and FWHM, one can distinguish an isolate signal of the diamond deposited on seed particles. In Figs. 2, we observe the seed particles covered with the deposited materials, and in Fig. 4, the diamond crystal peak at lower frequency position with broad FWHM are detected. Furthermore, Gand D-bands signals are undetectably small. Therefore, we consider that diamond crystal growth takes place on the seed particles in our system.

Knowing that the HFCVD works well for diamond growth on seed particles in the pressure rage of $100 \mathrm{~Pa}$, for the next step, we confirm the particles levitation in the rf plasma chamber equipped with a hot filament.

\subsection{Diamond particles levitation and a thermophoretic effect}

Fine particles levitate at the position where several forces are balanced. The forces are gravity, electrostatic, thermophoretic, ion drag, and others [1, 2]. In high substrate temperature range of $1000{ }^{\circ} \mathrm{C}$, the thermophoretic effect is particularly strong. Thus, one needs to control heating process in order not to disturb levitation.

The balance of the forces is described bellow.

$m_{p} g=Q E+F_{T}+\Sigma_{\mathrm{i}} F_{\mathrm{i}}$,

where $m_{p}$ is a mass of a particle, $Q$ is particle's charge, $F_{T}$ is a thermophoretic force and $\Sigma_{i}$ 
$F_{i}$ is a summation of other forces. Here, the thermophoretic force $F_{T}$ is given by the following equation [1]:

$F_{T}=-\left(8 r_{p}^{2} / 3 v\right) \Lambda(\mathrm{d} T / \mathrm{d} x)$,

where $\mathrm{d} T / \mathrm{d} x$ represents temperature gradient, $r_{p}$ is a particle radius and $v=(8 \mathrm{kT} / \pi \mathrm{m})^{1 / 2}$ is an average thermal velocity of gas atoms ( $k$ : Boltzmann factor, $T$ : gas temperature, $m$ : mass). $\Lambda$ is a coefficient of heat conductivity, and its detailed explanation is given in the literature [1]. According to the above equations, the forces balancing position (particle levitation position) changes when the temperature gradient $\mathrm{d} T / \mathrm{d} x$ changes. Indeed, we observe this phenomenon in our system. When the bottom electrode is heated up to a high temperature, the particles cloud position moves up. In most cases, the particles clouds finally disappear at higher substrate (bottom electrode) temperature than $100{ }^{\circ} \mathrm{C}$. On the other hand, it is possible to compensate this effect by heating the top electrode. The relation between the electrodes temperatures and levitation is summarized in Table 1. The experiments were carried out without a hot filament in order to observe the thermophoretic effects with a simple configuration. The results show that a compensation of thermophoretic effect is necessary for successful particles levitation.

\subsection{Particles levitation with a hot filament}

Since diamond growth using a HFCVD technique is typically carried out at a substrate temperature of $800{ }^{\circ} \mathrm{C}$, we also confirm the levitation in this temperature range. In this experiment, a hot filament was installed in the system. The levitation conditions are: bottom electrode $=800{ }^{\circ} \mathrm{C}$, top electrode $=800{ }^{\circ} \mathrm{C}$, filament temperature $=2000{ }^{\circ} \mathrm{C}$, rf input power $=50 \mathrm{~W}, \mathrm{H}_{2}=30 \mathrm{sccm}, \mathrm{He}=1 \mathrm{sccm}$ and total pressure $=50 \mathrm{~Pa}$. Note that, we use $\mathrm{Al}_{2} \mathrm{O}_{3}$ particles of diameter and mass density similar to those of diamond fine particles. Figure 5 shows the levitated particles at the above conditions that are equivalent to those for diamond growth. The top electrode heating is still required, although there is the 2000 ${ }^{\circ} \mathrm{C}$ hot filament above the bottom electrode, which supposes to compensate thermophoretic effect. 


\section{Conclusion}

We have observed diamond nucleation on seed particles placed on a Si substrate using the hot filament CVD. In order to obtain sufficient number of nucleation sites, a hydrogen pre-treatment procedure on the seed particles surfaces is necessary. Particles levitated in the plasma are particularly sensitive to thermophoresis due to inhomogeneities in the gas heating. Therefore, a proper heating procedure is necessary for successful particles levitation. We report the levitation of $\mathrm{Al}_{2} \mathrm{O}_{3}$ fine particles in $\mathrm{H}_{2} / \mathrm{He} \mathrm{rf}$ plasma equipped with a $2000{ }^{\circ} \mathrm{C}$ hot filament.

\section{Acknowledgement}

The authors would like to thank Dr. F. Jamitzky (MPE) for the use of the micro-Raman spectroscopy system at the Ludwig-Maximilians University Munich, Dr. C. Linsmeier (IPP) for the XPS measurement and useful discussion, Dr. S. Lindig (IPP) for the SEM measurements, Mr. B. Plöckl (IPP), Mr. T. Dürbeck (IPP) and Mr. V. Yaroshenko (MPE) for their technical support.

(MPE: Max-Planck-Institut für extraterrestrische Physik, IPP: Max-Planck-Institut für Plasmaphysik)

\section{References}

[1] H. Rothermel, T. Hagl, G. E. Morfill, M. H. Thoma, H. M. Thomas, Phys. Rev. Lett. 89 (2002) 175001.

[2] C. Zafiu, A. Melzer, A. Piel, Phys. Plasmas 9 (2002) 4794.

[3] H. Thomas, G. E. Morfill, V. Demmel, J. Goree, B. Feuerbacher, D. Möhlmann, Phys. Rev. Lett. 73 (1994) 652.

[4] B. M. Annaratone, G. E. Morfill, J. Phys. D: Appl. Phys. 36 (2003) 2853.

[5] G. Nishimura, S. Iizuka, T. Shimizu, N. Sato, Diamond. Relat. Mater. 12 (2003) 374.

[6] T. Shimizu, W. Jacob, H. Thomas, G. Morfill, T. Abe, Y. Watanabe, N. Sato, Thin Solid Films 506-507 (2006) 652.

[7] E. Kondoh, K. Tanaka, T. Ohta, J. Appl. Phys. 74 (1993) 2030. 
[8] L. L. Connell, J. W. Fleming, H.-N. Chu, D. J. Vestyck, Jr., E. Jensen, J. E. Butler, J. Appl. Phys. 78 (1995) 3622.

[9] Kuei-Hsien Chen, Mei-Chen Chuang, C. Murray Penney, W. F. Banholzer, J. Appl. Phys. 71 (1992) 1485.

[10] M. A. Childs, K. L. Menningen, L. W. Anderson, J. E. Lawler, J. Chem. Phys. 104 (1996) 9111.

[11] D. G. Goodwin, J. Appl. Phys. 74 (1993) 6888.

[12] J. E. Butler, R. L. Woodin, Phil. Trans. R. Soc. A 342 (1993) 209.

[13] C. H. Goeting, F. Marken, A. Gutiérrez-Sosa, R. G. Compton, J. S. Foord, Diamond Relat. Mater. 9 (2000) 390.

[14] Y. Kaenel, J. Stiegler, J. Michler, E. Blank, J. Appl. Phys. 81 (1997) 1726.

[15] J. W. Ager III, D. K. Veirs, G. M. Rosenblatt, Phys. Rev. B 43 (1991) 6491.

[16] T. A. Nachal'naya, V. D. Andreyev, E. V. Gabrusenok, Diamond Relat. Mater. 3 (1994) 1325.

\begin{tabular}{|c|c|c|c|c|}
\hline run \# & $\begin{array}{c}\text { rf power } \\
\text { W }\end{array}$ & $\begin{array}{c}\text { bottom electrode } \\
{ }^{\circ} \mathrm{C} \\
\end{array}$ & $\begin{array}{l}\text { top electrode } \\
{ }^{\circ} \mathrm{C} \\
\end{array}$ & levitation \\
\hline 1 & 30 & RT & $\mathrm{RT}$ & 0 \\
\hline 2 & 30 & 70 & $\mathrm{RT}$ & $\times$ \\
\hline 3 & 30 & 70 & 260 & O \\
\hline 4 & 70 & RT & RT & O \\
\hline 5 & 70 & 190 & 100 & $\times$ \\
\hline 6 & 70 & 190 & 380 & 0 \\
\hline
\end{tabular}



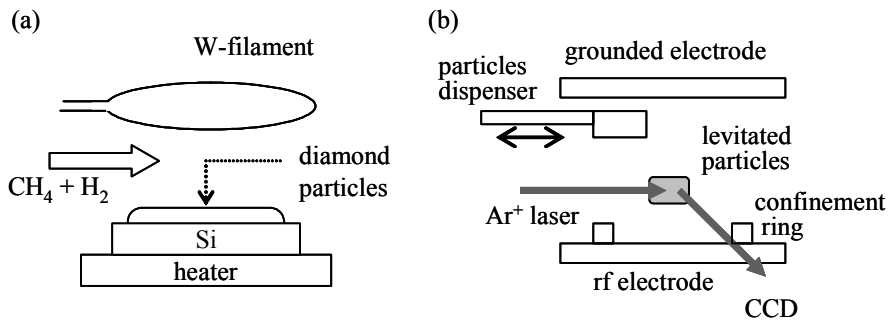

Fig. 1. (a) The HFCVD system used for the diamond growth. (b) The rf plasma system used for the particles levitation.

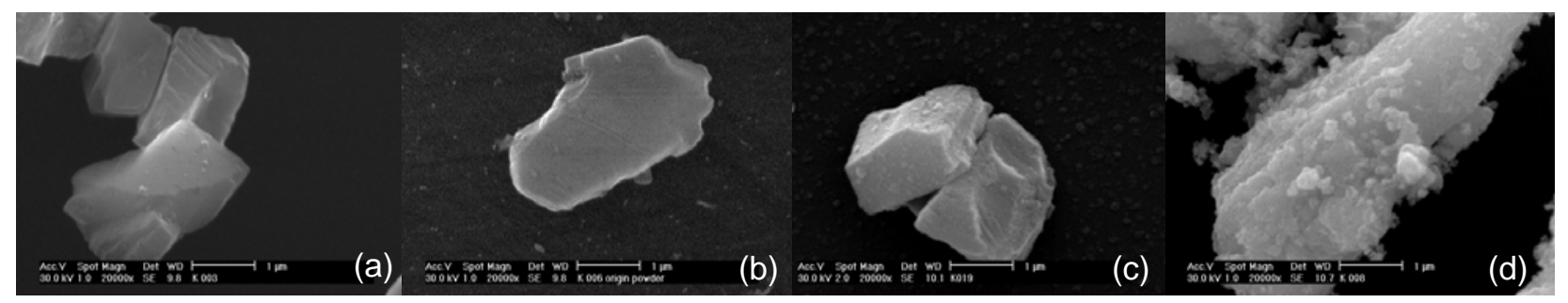

Fig. 2. The SEM images of the diamond particles. (a) seed particles without deposition. (b) - (d) diamond deposited samples with different hydrogen pre-treatment time ((b) 0 h, (c) 1 $\mathrm{h}$ and (d) $3 \mathrm{~h}$ ). 


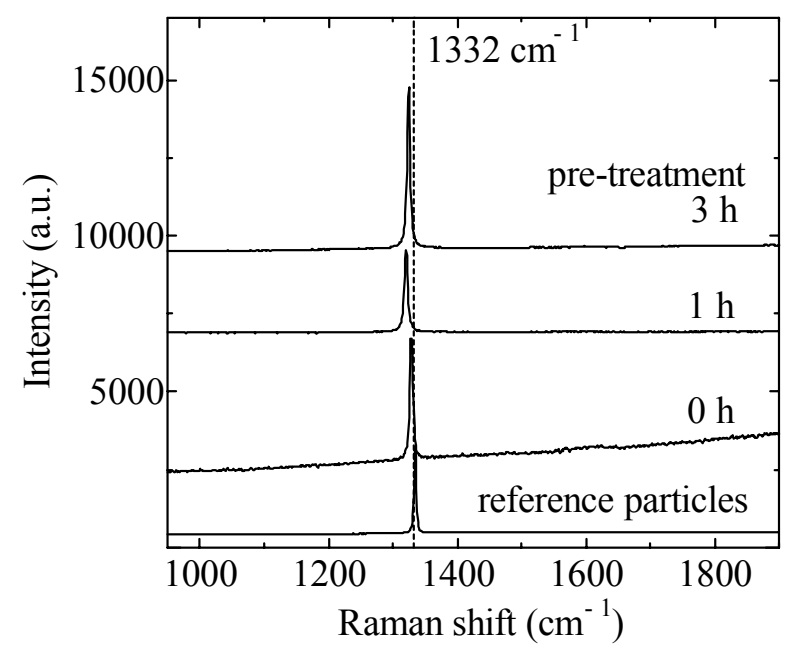

Fig. 3. The micro-Raman spectra of the samples prepared with the hydrogen pre-treatment time of 0,1 , and $3 \mathrm{~h}$. The spectrum of the seed particles without deposition is also shown.

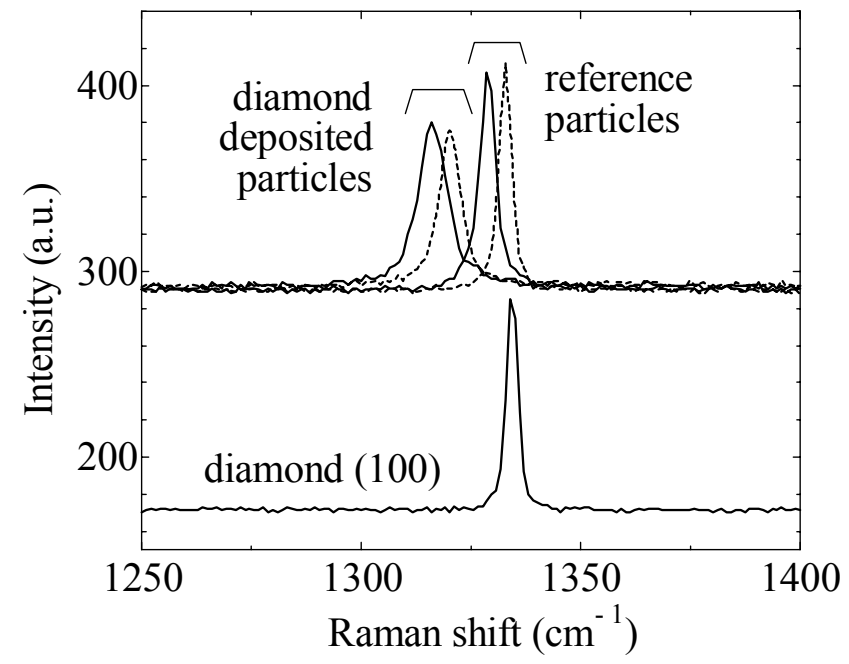

Fig. 4. The micro-Raman spectra of the seed particles and the diamond deposited sample (with the hydrogen pre-treatment time of $3 \mathrm{~h}$ ). The spectrum of the diamond (100) substrate is also shown. 


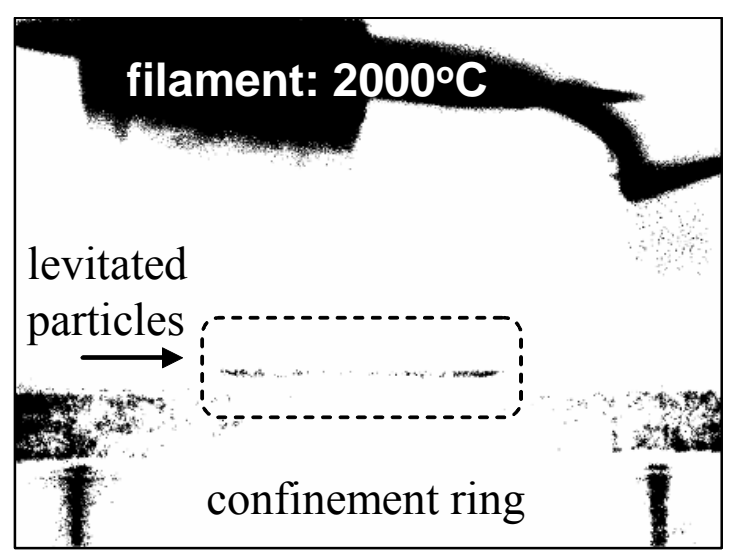

Fig. 5. The pictures of the levitated $\mathrm{Al}_{2} \mathrm{O}_{3}$ particles in the rf plasma equipped with a hot filament. The picture colors are inverted for easier view of particles. 